\title{
Gastroenteritis aguda causada por Vibrio cholerae no-O1, no-O139 que porta una región homóloga a la isla de patogenicidad VpaI-7
}

\author{
María T. Ulloa F., Lorena Porte T., Stephanie Braun J., Jeannette Dabanch P., \\ Alberto Fica C., Tania Henríquez A. y Carlos G. Osorio A.
}

\begin{abstract}
Hospital Militar, Santiago, Chile (LPT, SBJ, JDP, AFC) Universidad de Chile, Santiago, Chile. Facultad de Medicina, Instituto de Ciencias Biomédicas Programa de Microbiología y Micología (MTUF, THA, CGOA)

Los autores declaran no tener conflictos de interés.

Financiamiento: Proyecto InnovaCorfo 09CN14-5951.

Recibido: 25 de octubre de 2010 Aceptado: 14 de abril de 2011

Correspondencia a: Carlos G Osorio A gonosorio@med.uchile.cl
\end{abstract}

\section{Acute gastroenteritis caused by a Vibrio cholerae non-O1, non-O139 strain harboring a genetic region homologous to the VpaI-7 pathogenicity island}

Pathogenic Vibrio cholerae isolates, the etiologic agents of cholera, generally express one of two O antigens (O1 or 0139). Most environmental isolates are nonpathogenic and are referred to as "non-O1, non-O139". However some $V$. cholerae non-O1, non-O139 strains are clearly pathogenic and have caused outbreaks or sporadic cases of gastroenteritis and extraintestinal infections in humans. We report a case of acute gastroenteritis by a V. cholerae non-O1, non-O139 harboring a genetic region homologous to a segment of the VpaI-7 V. parahaemolyticus pathogenicity island.

Key words: Gastroenteritis, Vibrio cholerae, non-O1, non-O139, pathogenicity island.

Palabras clave: Gastroenteritis, Vibrio cholerae, no-O1, no-O139, isla de patogenicidad.

\section{Introducción}

L a bacteria gramnegativa Vibrio cholerae es el agente etiológico del cólera, un tipo de diarrea secretora que puede causar deshidratación grave y tener alta letalidad. La mayoría de las cepas patogénicas de $V$. cholerae poseen en su membrana externa un antígeno denominado O, porción más externa del lipopolisacárido (LPS), del tipo O1 u O139. La mayoría de las cepas ambientales de esta bacteria presenta otros tipos de antígeno O y por eso se categorizan como no-O1, no-O1391-4. Sin embargo, algunas cepas de $V$. cholerae no-O1, no-O139 poseen un claro poder patógeno y han causado brotes o casos esporádicos de gastroenteritis e infecciones extraintestinales en humanos ${ }^{5-7}$.

La transmisión de $V$. cholerae no-O1 no-O139 se relaciona con el consumo de alimentos de origen marino, especialmente mariscos bivalvos crudos. Las infecciones en humanos causadas por $V$. cholerae no-O1, no-139 muestran variadas presentaciones clínicas; la más frecuente corresponde a un cuadro de gastroenteritis aguda con diferentes grados de gravedad. Una forma bastante infrecuente de esta infección es la septicemia ${ }^{5,6}$. Los casos reportados de septicemia se asocian generalmente con inmunosupresión y/o daño hepático crónico. Unos pocos casos de asocian a infección cutánea primaria.

Los factores de virulencia que expresan las cepas de $V$. cholerae no-O1, no-O139 prácticamente se desconocen.
Un trabajo realizado por el laboratorio de Mekalanos y cols., en el año 2005, describió por primera vez una cepa clínica de $V$. cholerae no-O1, no-O139 que porta en su genoma un segmento homólogo de la isla de patogenicidad denominada VPaI-7 de V. parahaemolyticus ${ }^{7}$. Este segmento génico codifica para un sistema de secreción tipo III (TTSS) funcional que probablemente pueda estar contribuyendo a la virulencia de este tipo de cepas $^{8,9}$.

Este trabajo describe por primera vez en Chile un caso de gastroenteritis aguda cuyo agente etiológico corresponde a una cepa de $V$. cholerae no-O1, no-O139 que porta una estructura genética similar a la descrita previamente por el grupo de Mekalanos.

\section{Caso clínico}

Mujer de 63 años con antecedentes de hipertensión arterial, intolerancia a la glucosa, dislipidemia en tratamiento y un episodio de diverticulitis aguda no complicada en el año 2004. A comienzos de enero de 2009, la paciente inició un cuadro caracterizado por deposiciones líquidas abundantes y explosivas con mucus, sin sangre ni pus, asociadas a dolor abdominal tipo cólico y fiebre de hasta $39{ }^{\circ} \mathrm{C}$ con calofríos. Por persistencia de los síntomas, consultó al segundo día de evolución en un centro privado de salud donde se le realizó una tomografía axial computarizada de abdomen, que demostró signos 
compatibles con inflamación de íleon terminal, colon ascendente y transverso. Sólo se le indicó tratamiento sintomático. Por persistencia de diarrea grave de alta frecuencia y dolor abdominal asociado a vómitos, al tercer día de evolución, consultó al Servicio de Urgencia del Hospital Militar de Santiago, donde se decidió su internación con el diagnóstico de colitis infecciosa y deshidratación leve. Al examen físico de ingreso se constató una temperatura axilar de $37,4{ }^{\circ} \mathrm{C}$, taquicardia con frecuencia de $113 \mathrm{x}$ ', presión arterial normal, signos de deshidratación leve y abdomen distendido, sensible en forma difusa y con ruidos hidroaéreos aumentados. Se solicitaron exámenes de laboratorio incluidos hemograma, hemocultivos, electrolitos plasmáticos, proteína $\mathrm{C}$ reactiva (PCR), glicemia y estudio de deposiciones (leucocitos fecales, coprocultivo, detección de rotavirus y adenovirus entérico). En los resultados sólo destacó una PCR elevada de 44,25 mg/dL (VN:0-1), hipokalemia de 3,11 mEq/L (VN: 3,5-5,3), glicemia discretamente elevada de $156 \mathrm{mg} / \mathrm{dL}$ (VN:70-99) y leucocitos fecales $>20$ por campo. A las 24 horas de ingresar, se inició tratamiento con ceftriaxona y metronidazol, hidratación parenteral y corrección de la hipokalemia. El cuadro febril cedió, pero pese al tratamiento, la paciente persistió con diarrea intensa, entre 8-10 deposiciones/día, dolor abdominal y vómitos. Se obtuvo, además, el antecedente de tres personas relacionadas a la paciente que habrían presentado síntomas gastrointestinales similares, sin lograr más información. En el coprocultivo tomado al ingreso hubo desarrollo de un bacilo gramnegativo a las 72 horas de incubación, identificado como Vibrio spp. Los cultivos en agar TCBS y Chromoagar, la prueba de la oxidasa y la identificación por Microscan fueron concordantes con el diagnóstico presuntivo de $V$. cholerae. El aislado fue derivado al Programa de Microbiología del Instituto de Ciencias Biomédicas (ICBM) de la Universidad de Chile para su caracterización molecular. Se modificó el tratamiento antimicrobiano, continuando con ciprofloxacina 500 mg cada 12 horas por vía oral. Los hemocultivos fueron negativos al séptimo día. A los 10 días de evolución, la paciente presentó franca mejoría, cesando los vómitos y disminuyendo la frecuencia de las deposiciones. Completó ocho días de tratamiento antimicrobiano y fue dada de alta en buenas condiciones a los 11 días de evolución.

\section{Caracterización molecular}

Con el objetivo de profundizar en la caracterización de esta nueva cepa clínica de V. cholerae no-O1, noO139, ésta fue derivada al Laboratorio de Microbiología Molecular de Vibrios del Programa de Microbiología y Micología de la Facultad de Medicina de la Universidad de Chile. Estudios de serotipificación con antisueros antiO1 y anti-O139 demostraron que este aislado pertenece al tipo no-O1 y no-O139. Todos los partidores utilizados en este estudio para ensayos de reacción de polimerasa en cadena (RPC) se detallan en la Tabla 1. Con el fin de corroborar los resultados serológicos se amplificó y secuenció una región del gen del ARN 16S utilizando el par de partidores: fD1 y rP2 ${ }^{10}$. La secuencia obtenida (Laboratorio Dr. Andrew Camilli, Tufts University Medical School, Boston, MA, USA) fue analizada por BLAST confirmando con certeza que la secuencia pertenece a la especie $V$. cholerae (el mayor grado de identidad, esto es 99\%, ocurrió con la cepa de V. cholerae AM-19226 que pertenece al tipo no-O1, no-O139). Entre los probables genes de virulencia de esta cepa se estudiaron por RPC estándar la toxina del cólera (CTX), el pilus TCP y la presencia de un segmento homólogo de la isla de patogenicidad denominada VPaI-7 de V. parahaemolyticus ${ }^{4-7}$. El gen $c t x A$ y tcpA codificantes para la subunidad catalítica de la CTX y la subunidad pilina del pilus TCP, respectivamente, resultaron ambos negativos. Se realizaron además ensayos de RPC estándar para amplificar diferentes segmentos, incluyendo ambos extremos y la zona central, de la región génica descrita previamente por el grupo de Mekalanos ${ }^{7}$. El objetivo de este ensayo fue establecer con cierto grado de confidencia si nuestra cepa diarreogénica portaba esta estructura genética de manera completa o parcial. Para ello, los siguientes pares de partidores fueron utilizados: el par P5 fue diseñado para amplificar el segmento proximal de la isla de patogenicidad, el par Pm para la región central y el par P3 para su región terminal (Tabla 1, Figura 1). Los pares P5 y Pm amplificaron un fragmento del tamaño esperado. El par P3 amplificó un

Tabla 1. Partidores usados en la caracterización de la región genómica que presenta homología con la isla de patogenicidad Vpal-7 de Vibrio parahaemoIyticus en cepas clínicas de Vibrio cholerae no-01, no-0139a

\begin{tabular}{llcl}
\hline Partidor & Secuencia 5'-3' & Producto (bp) & Referencia \\
CTX-F & CGGGCAGATTCTAGACCTCCTG & 564 & $(9)$ \\
CTX-R & CGATGATCTTGGAGCATTCCCAC & & \\
TCPA-F & AGCCGCCTAGATAGTCTGTG & 1.324 & $(4)$ \\
TCPA-R & TCGCCTCCAATAATCCGAC & & \\
fD1 & AGAGTTTGATCCTGGCTCAG & 1.500 & $(8)$ \\
rP2 & ACGGCTACCTTGTTACGACTT & & \\
P5f & CTTCATATTCAACACCTTC & 2.500 & Estudio actual \\
P5r & CTATGCTGTTTAACCTTTG & & Estudio actual \\
Pmf & TAACTGATACAACTGACTAC & & Estudio actual \\
Pmr & TAACTGGTTGACTCGATC & Estudio actual \\
P3f & GTTCTCTGCGATATTCACTAATG & 1.832 & Estudio actual \\
P3r & CATTTTCATTGACCACGAC & Estudio actual \\
aTodos los partidores usados en este trabajo se diseñaron en base a la secuencia completa de la isla de \\
patogenicidad DQ124262 (7) y de secuencias obtenidas en nuestro laboratorio.
\end{tabular}




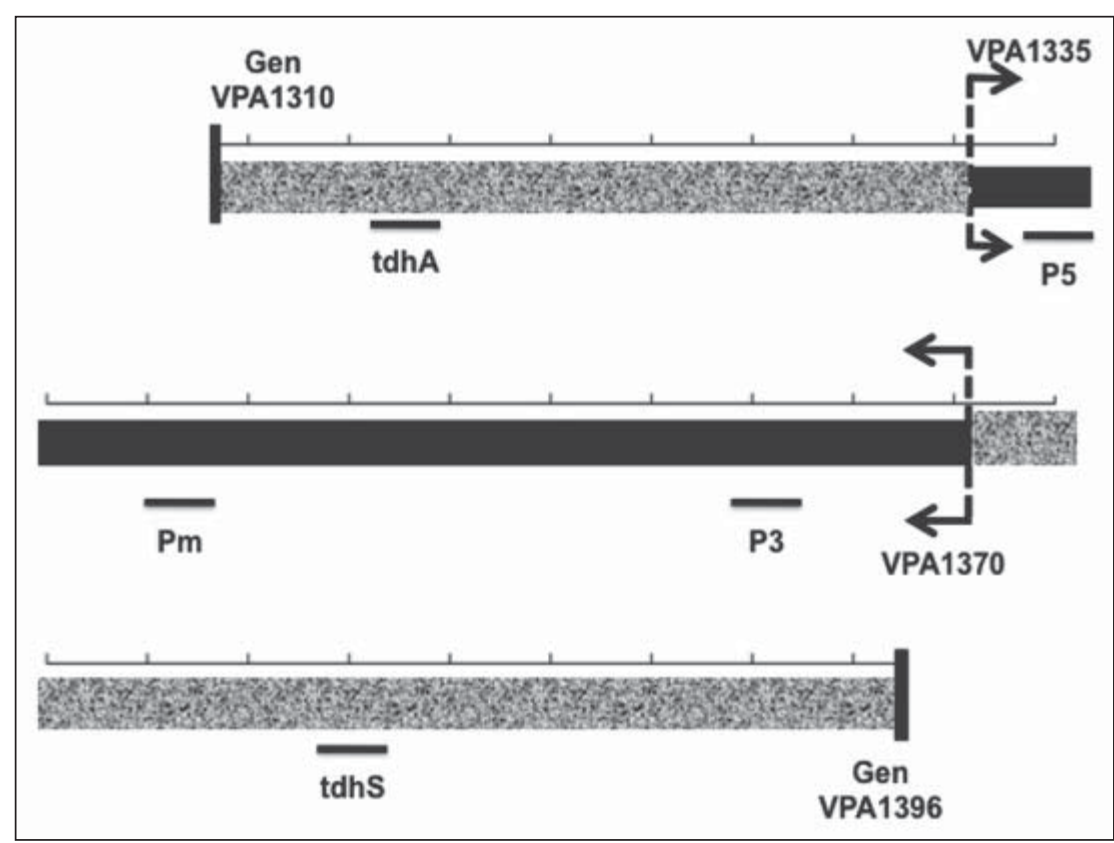

Figura 1. Esquema del segmento homólogo a isla de patogenicidad Vpal-7 de $V$. parahaemolyticus detectado recientemente en cepa de $V$. cholerae no-O1, no-O139 (7). La isla de patogenicidad original de V. parahaemolyticus, denominada Vpal-7, se extiende entre los genes VPA1310 y VPA-1396 (indicados en ambos extremos del esquema). La zona detectada en $V$. cholerae no-O1, no-O139 abarca aproximadamente la región entre los genes VPA1335 y VPA1370 (se acota con líneas punteadas y bloque de color negro). Los extremos de la isla Vpal-7, no presentes en $V$. cholerae no-O1, no-0139 se destacan en bloques con textura gris. Los pares de partidores utilizados para detectar esta isla se indican con segmentos de línea horizontal como P5, Pm y P3. Se indican como referencia los genes tdhA y tdhS de la isla Vpal-7 de V. parahaemolyticus.

Figura 2. RPC estándar de segmento homólogo a isla de patogenicidad Vpal-7 de V. parahaemolyticus presente en cepa diarreogénica de $V$. cholerae no-O1, no-O139. Los siguientes pares de partidores fueron utilizados: P5 (tamaño esperado: aprox. $2.500 \mathrm{pb}$; carril 1); Pm (tamaño esperado: $873 \mathrm{pb}$; carril 2); $\mathrm{P3}$ (tamaño esperado: $1.832 \mathrm{pb;}$ carril 3). En ambos extremos se muestran marcadores de peso molecular de $1 \mathrm{kpb}$ GeneRuler (ver detalles en el texto principal). segmento de un tamaño de aproximadamente $3.000 \mathrm{pb}$ siendo el tamaño esperado 1.832 pb (Figura 2). Dada la diferencia de tamaños, el amplicón P3 fue secuenciado parcialmente para corroborar su identidad.

\section{Discusión}

En este trabajo se ha logrado caracterizar microbiológica y molecularmente una cepa clínica de la especie $V$. cholerae, perteneciente al serotipo no-O1, no-O139. En su gran mayoría, este tipo de cepas carece de los principales factores de virulencia presentes en $V$. cholerae $01 / 0139$, tales como la toxina del cólera (CTX) y el pilus tipo IV denominado $\mathrm{TCP}^{2,3,11}$. Sin embargo, algunos reportes recientes indican que estas cepas podrían compartir regiones de la isla de patogenicidad genómica denominada VpaI-7 de la especie $V$. parahaemolyticus ${ }^{7}$. La isla de patogenicidad VpaI-7 porta los principales genes de virulencia de este vibrión (gen $t d h$ que codifica la hemolisina directa termoestable o TDH) y otros probables genes de virulencia (conocidos en inglés como putative virulence genes) ${ }^{12}$. Fue así como nuestro laboratorio pudo describir una cepa clínica septicémica de $V$. cholerae no-O1, no-O139 que portaba el gen $t d h^{5}$.

En este trabajo se presenta un nuevo aislado de $V$. cholerae no-O1, no-O139, proveniente de un caso de gastroenteritis aguda y que porta una estructura genética homóloga a la descrita previamente por el grupo de Mekalanos ${ }^{7}$. Los amplicones P5 y Pm presentaron un tamaño esperado. El amplicón P3 correspondió a un segmento de aprox $3.000 \mathrm{pb}$, siendo el tamaño esperado 1.832 pb. Dada la diferencia de tamaños, este amplicón fue parcialmente secuenciado confirmándose su correspondencia con el segmento P3 previamente descrito. La diferencia de tamaños se explica por una inserción, aún no caracterizada, presente en el locus P3 de nuestra cepa diarreogénica. En breve, la confirmación de la presencia de estos tres segmentos génicos (P5, Pm y P3) en nuestra cepa indica que ésta probablemente porta la estructura completa descrita originalmente ${ }^{7}$.

La información obtenida, junto con los datos de la literatura científica, permiten plantear la posibilidad de que un porcentaje mínimo de las cepas ambientales de $V$. cholerae no-O1, no-O139 haya captado e incorporado en el curso de la evolución trozos de la isla de patogenicidad de V.parahaemolyticus, y que este evento de transferencia genética horizontal las haya transformado en cepas patogénas para el ser humano. Sin embargo, dado el escaso número de cepas estudiadas hasta el momento, no es aún posible realizar inferencias precisas respecto a la importancia de este elemento genético. Actualmente estamos recolectando activamente cepas clínicas y ambientales de $V$. cholerae no-O1, no-O139 con el fin de determinar si esta región genética se concentra preferentemente en las cepas 
de tipo clínico respecto de las ambientales. Paralelamente, el estudio de mutantes nulas para esta región genética en modelos in vivo permitirá establecer con certeza el papel que esta estructura genética desempeña en virulencia. Es importante destacar que este tipo de cepas de $V$. cholerae se ha visto cada vez más activamente involucrado en diversos casos clínicos reportados en nuestro país: un caso de septicemia fatal en Valparaíso en $2006^{5}$, 15 aislados clínicos reportados en todo el país en 2009 por el Instituto de Salud Pública (ISP) ${ }^{13}$, una cepa clínica reportada en Santiago en 2009 (presente estudio) y seis aislados clínicos confirmados en un brote en Antofagasta 2010 por ISP ${ }^{13}$. Estos antecedentes demuestran la importancia clínica de este tipo de cepas de $V$. cholerae en nuestro país. Conjugando estos datos epidemiológicos con una probable sub-notificación de estos microorganismos en nuestro país, pone en evidencia la urgente necesidad de estudiar detalladamente la epidemiología y el potencial patogénico de V. cholerae no-O1, no-O139.
Agradecimientos. Al académico del Programa de Microbiología del ICBM, Germán Hermosilla, por su valioso aporte en la revisión y discusión de este trabajo.

\section{Resumen}

Cepas patogénicas de Vibrio cholerae, el agente causal del cólera, expresan generalmente uno de dos antígenos O (denominados 01 u O139). La mayoría de las cepas ambientales son no patogénicas y corresponden al tipo denominado "no-O1, no-O139". Sin embargo, algunas cepas de este tipo son claramente patogénas y han causado brotes de gastroenteritis e infecciones extra-intestinales en humanos. Se reporta un caso clínico de gastroenteritis aguda causado por una cepa de V. cholerae no-O1, noO139 que contiene en su genoma una región homóloga a un segmento de la isla de patogenicidad VpaI-7 descrita previamente en $V$. parahaemolyticus.

\section{Referencias}

1.- Bidinost C, Saka H, Aliendro O, Sola C, Panzetta-Duttari G, Carranza P, et al. Virulence factors of non-O1 non-O139 Vibrio cholerae isolated in Córdoba, Argentina. Rev Argent Microbiol 2004; 36: 158-63.

2.- $\quad$ Faruque S M, Albert M J, Mekalanos J J. Epidemiology, genetics, and ecology of toxigenic Vibrio cholerae. Microbiol Mol Biol Rev 1998; 62: 1301-14.

3.- Thompson F L, Iida T, Swings J. Biodiversity of Vibrios. Microbiol Mol Biol Rev 2004; 68: 403-31.

4.- $\quad$ Boyd E F, Waldor M K. Evolutionary and functional analyses of variants of the toxincoregulated pilus protein TcpA from toxigenic Vibrio cholerae non-O1/ non-O139 serogroup isolates. Microbiology 2002; 148: 1655-66.

5.- Briceño I, Puebla C, Guerra F, Jensen D, Núñez H, Ulloa M T, et al. Primer caso septicemia fatal causada por Vibrio cholerae no-O1, no-O139 hemolítico en Chile. Rev Med Chile 2009; 137: 1193-6.

6.- Halabi M, Haditsch M, Renner F, Brinninger G, Mitter-Mayer H. Vibrio cholerae non-O1 septicaemia in a patient with liver cirrhosis and Billroth-II-gastrectomy. J Infect 1997; 34: 83-4.

7.- Dziejman M, Serruto D, Tam VC, Sturtevant D, Diraphat P, Faruque SM, et al. Genomic characterization of non-O1, non-O139 Vibrio cholerae reveals genes for a type III secretion system. Proc Natl Acad Sci USA 2005; 102: 3465-70.

8.- Tam V C, Serruto D, Dziejman M, Brieher W, Mekalanos J J. A type III secretion system in Vibrio cholerae translocates a formin/spire hybrid-like actin nucleator to promote intestinal colonization. Cell-Host Microbe 2007; 1: 95107.

9.- Alam A, Tam V, Hamilton E, Dziejman M. vttRA and vttRB encode ToxR family proteins that mediate bile-induced expression of type three secretion system genes in a non-O1/ non-O139 Vibrio cholerae strain. Infect Immun 2010; 78: 2554-70.

10.- Weisburg W G, Barns S M, Pelletier D A, Lane D J. 16S ribosomal DNA amplification for phylogenetic study. J Bacteriol 1991; 173 : 697-703.

11.- Fields P, Popovic T, Wachsmuth K, Olsvik O. Use of polymerase chain reaction for detection of toxigenic Vibrio cholerae $\mathrm{O} 1$ strains from the Latin American cholera epidemic. J Clin Microbiol 1992; 30: 2118-121.

12.- Makino K, Oshima K, Kurokawa K, Yokoyama K, Uda T, Tagomori K, et al. Genome sequence of Vibrio parahaemolyticus: a pathogenic mechanism distinct from that of $V$. cholerae. Lancet 2003; 361:743-9.

13.- Comunicados epidemiológicos Instituto de Salud Pública de Chile. 2010. 6 y 7 de abril de 2010. 